\title{
Back to the future: A review of forty years of population projections at Statistics Canada
}

\author{
Patrice Dion ${ }^{1}$ \\ Nora Galbraith
}

\begin{abstract}
This paper aims to provide an overview of the population projections program at Statistics Canada, including its orientation, its strengths and challenges. We first identify some conceptual issues which have a bearing on how projections should be interpreted and evaluated. Then, we briefly review the past editions of Statistics Canada's population projections and identify their main strengths and limitations. The evaluation considers the performance of previous projections at the national and provincial/territorial geographic levels and in terms of each of the major components of growth (fertility, mortality, and migration).
\end{abstract}

Keywords: Population projections, forecast, evaluation, Statistics Canada, accuracy.

\section{Résumé}

Cet article vise à fournir une vue d'ensemble du programme des projections démographiques de Statistique Canada, incluant son orientation, ses forces et ses défis. Nous relevons d'abord certains enjeux conceptuels ayant des répercussions sur la façon dont les projections devraient être interprétées et évaluées. Nous jetons ensuite un bref coup d'œil aux éditions passées des projections de Statistique Canada et identifions leurs points forts et leurs points faibles. Notre évaluation comprend la performance des projections à l'échelon national et à celui des provinces et des territoires, ainsi qu'à l'égard des diverses composantes de la croissance démographique (fécondité, mortalité et immigration).

Mots-clés: Projections de population, prévisions, évaluation, Statistique Canada, exactitude.

\section{Introduction}

The year 2014 marked 40 years since the first official release of population projections by Statistics Canada, the 1972-2000 edition. It also marked the release of the ninth edition of the projection, for the period 2013-2063. Between these editions, many developments occurred, both in the methodology of the projections and in the demographic context of Canadian society.

1. Patrice Dion, Manager, Demographic Analysis and Projections Section, Demography Division, Statistics Canada, 150 Tunney’s Pasture Driveway, SC 1708 I, Ottawa ON K1A 0T6. Email: patrice.dion@statcan.gc.ca; Nora Galbraith, Analyst, Demographic Analysis and Projections Section, Demography Division, Statistics Canada, Ottawa. 
This article provides an overview of the evolution of the National Population Projections program at Statistics Canada, including its orientation, its strengths and challenges, and recent improvements and developments. We initiate the discussion with a reflection on what characterizes a population projection, and how this in turn defines what we should expect from it. We then proceed to a brief evaluation of past projections made by Statistics Canada before concluding.

\section{Evaluation of population projections}

At Statistics Canada, the publication of population projections must conform to certain standards and policies, including those established in the agency's own internal Policy on Estimates with Future Reference Dates. This policy states, among other things, that there should be several estimates for any given future reference date, each being the product of different and clearly stated assumptions, or the product of alternative specifications of the model. The policy also states that no single set of estimates should be labeled as "most probable." In this context, it is clear that Statistics Canada publishes projections, not forecasts.

This precision relates to the distinction between projections and forecasts. Projections and forecasts draw from two distinct approaches, behind each of which stands a theory, or epistemological stance, regarding our knowledge of the future (Romaniuc 2010). As Lachapelle (1977) explains, projections aim to retain all assumptions that seem plausible, while forecasts use only those that are the most likely. Thus, while a forecast will attempt to predict what will occur in the future, a projection seeks to show the outcome of a set of assumptions if they happened to be realized (Keyfitz and Caswell 2005). As a result, a forecast could eventually be evaluated in light of future events, while a projection will be proven wrong only if an error in the underlying calculation is found (George et al. 2004).

However, as clear as the distinction between forecast and projection appears to be, the two may not be so easily distinguished in reality. This is because the reader most often considers the projection to be a forecast (Henry 1972). Moreover, even though more than one projection scenario exists, users almost invariably turn to the middle variant and interpret it as a forecast (Keyfitz 1981). Keyfitz (1972) questions what criteria should define a projection versus a forecast: is it the demographer's intention or the reader's use? He also argues that if demographers cannot judge which ranges of mortality or fertility are most likely to be realized, then no one can; in practice, users have depended on demographers for such judgment (Keyfitz 1981). De Beer adds that "To let users make their own choice does not seem an optimal use of expertise" (2000: 26).

Since they are likely to be used and interpreted as forecasts, attempts to produce projections rather than forecasts may be thought of by some as exercises in futility. In practice, demographers who use the label "projection" rather than "forecast" adopt a prudent position, a perspective that underlines the high level of uncertainty inherent to projection exercises. ${ }^{2}$ From the perspective of population projections (and not population "forecasts"), a plausible assumption is not one that will necessary materialize, but one that at the time of its conception suggested a reasonable and realistic evolution given the existing knowledge.

If, as stated earlier, population projections would be proven wrong only if an error in the underlying calculation is found - and not in light of future events-is the evaluation of accuracy ${ }^{3}$ totally irrelevant? In reality, demographers in the process of building projection assumptions find themselves wondering what outcomes are the most likely to occur. If this kind of process did not exist, there

2. They will also, traditionally, offer a variety of alternative outcomes in order to reflect this uncertainty and avoid attaching any sense of probability to the projection values.

3. The term "accuracy" in this article refers to correspondence between projected and observed values. 
would be no special value given to a projection, and one projection would be as good as any other (Keyfitz 1972). Therefore, the notion of predicting the future is not totally removed from population projections; rather, it is subtly embedded within the assumption-building process.

There are, in our opinion, at least three other reasons why accuracy, as measured against actual observed outcomes, plays an important role in population projections. Firstly, projections are informative of the period in which they were built; the examination of past projections" "errors" reveals both the continuity and the changes in demographic trends that have occurred since their release. ${ }^{4}$ Secondly, reflecting on the sources of past inaccuracies can serve as a basis for improving future projection assumptions and methodologies (Wilson and Rees 2005). Finally, repeated comparison of projected values with historical estimates informs us about the limitations of demographic projections and what we can reasonably expect from them.

Still, some precautionary statements should be given before attempting such an exercise. Accuracy is an important aspect of quality, but should not be the only criterion. In the end, whether a forecast or a projection, a crucial component of the evaluation is the utility of the exercise as perceived by its users (Romaniuc 2010). Results from surveys of the users of forecasts and projections suggest many other criteria should be part of an evaluation of a projection method or program. Particularly, the adoption of a given methodology based on its accuracy power should be weighed against other aspects, such as its ease of interpretation and use, timeliness, and cost (Yokum and Armstrong 1995; Rayer 2008). The main utility of projections for those who use them is as a tool to aid planning. Their usefulness is therefore not situated in the future, but in the very present. The analytical credibility awarded to the projections at the time of their release is therefore critical (Romaniuc 2003, 2010).

With these cautions in mind, we now proceed to a brief overview of Statistics Canada's population projections program, and an evaluation of the accuracy of past editions of its National Population Projections.

\section{Overview of Statistics Canada's past population projections}

Before the 1970s, Statistics Canada had prepared some ad-hoc projections, ${ }^{5}$ however, these were not given general distribution (George 2001). Beaujot (2000) notes that these projections had limited success in estimating the demographic growth of the country, not anticipating the major changes associated with the baby boom and the increase in international migration.

It was not until the 1970s that population projections became a regular activity at Statistics Canada. In recent years, Statistics Canada has produced official population projections through two distinct programs. The National Population Projections program is responsible for publishing national, provincial, and territorial population projections at regular intervals, usually every 5 years, following the census cycle. These projections, available by age and sex, utilize the cohort-component model, which projects each component of population growth separately. While some have questioned the "value added," in terms of projection accuracy, of the cohort-component method over extrapolation and "naïve" methods which are comparatively simpler and less costly to produce (Tayman and Swanson 1996; Rayer 2008), the cohort-component method holds two main advantages that have motivated its use by the agency. Firstly, the structure of the cohort-component method mirrors and

4. Projection results may themselves be a factor in subsequent changes (for instance, by highlighting a possible decline in the working-age population that could trigger a rise in immigration levels).

5. For example, a set of projections made by the Dominion Bureau of Statistics around 1950 were subject to restricted distribution for the given reason that "the calculations do not have the same factual basis as other Bureau publications" (Preston 1974: 720). 
thus extends the structure of the data produced by Statistics Canada's Population Estimates Program, upon which the projections are based. Another benefit of the cohort-component model is that it allows for the elaboration of specific assumptions that are consistent with the knowledge held for each (O'Neill et al. 2001). This benefit supports the production of a credible projection of not only the total population size but also the age structure of the population, though, as will be seen later, by no means guarantees its accuracy in this regard.

In the context of the National Population Projections program, Statistics Canada also produces, upon request and on a cost-recovery basis, custom population projections for various levels of geographies. Additionally, since 2005 Statistics Canada has published a series of population projections using a microsimulation approach, which projects the life events of each individual in the population. ${ }^{6}$ Also treating the components of population growth separately, these projections tend to use the same assumptions about the components as those used in the National Population Projections program. However, they also make assumptions about, and project, various individual characteristics such as education, marital status, visible minority status, Aboriginal identity, and labour force participation. These additional projection dimensions allow for a more detailed description of the future composition of the Canadian population under different scenarios of population growth. Given the time and resources needed for their development, projections using microsimulation models have, to date, been made upon request and with the financial assistance of external partners or stakeholders. Having considerably different parameters and context, as well as a much shorter history, an evaluation of the agency's microsimulation projections would require a framework distinct from that performed for the National Population Projections. In the remainder of the article, therefore, we will focus solely on the National Population Projections program of cohort-component-based projections, its past projections, and new developments.

\section{Projections at the national level}

Since the 1970s, Statistics Canada has published nine editions of population projections. In a demographic context that turned out to be much more stable than in the early post-war period (Beaujot 2000), these editions generally succeeded in projecting the demographic growth and the population aging that would follow at the national level.

With its time span now past, it is very instructive to evaluate how well the 1972-2001 edition did in terms of estimating the future population of Canada. Indeed, this edition did incredibly well at first glance: the medium-growth scenario suggested that the Canadian population would reach 31,050,000 in 2001, a number which ultimately overestimated reality by only 30,000, or less than 0.1 per cent (Figure 1). However, a closer look suggests that we should not attribute this "success" to correct assumptions, but instead to a series of compensatory errors. In fact, this scenario suggested that a total fertility rate (TFR) of 2.2 children per woman would be reached in 1985 and held constant thereafter, along with an annual admission of 120,000 immigrants. In reality, in 2001, the TFR was 1.54 children per woman and the number of immigrants was more than double that of the assumption. These divergences had important consequences: the age structure of the Canadian population in 2001 greatly differed from that which was projected for that year, with a much older population composition occurring in reality (Figure 2).

The range of the low, medium, and high projection scenarios in the 1976-2001 edition encompassed the observed population in 2001 by a very small margin, with the growth of the Canadian

6. See Statistics Canada $(2010,2011)$ for recent reports of projections made by the agency with microsimulation models. 


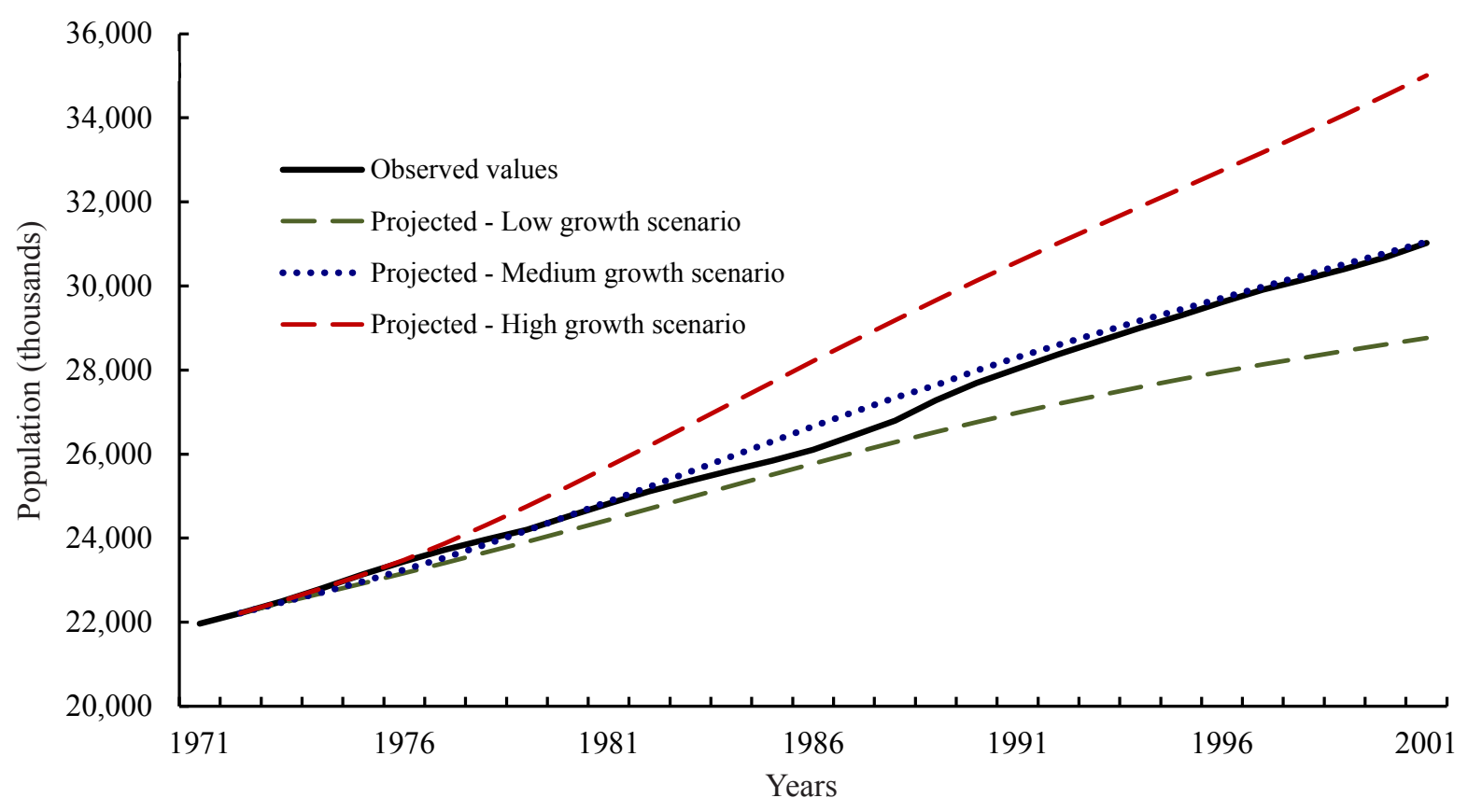

Figure 1. Population observed and projected according to three scenarios of the 1972-2001 edition, Canada. Source: Adapted from Statistics Canada, Demography Division. 2010. Population Projections for Canada, Provinces and Territories: 2009 to 2036. Chart 5.1.

Note: The projected figures were adjusted to take into account revisions made to the population estimates after the release of the projections. Essentially, these revisions consisted of adding net undercoverage and components that were not estimated previously such as non-permanent residents and returning emigrants.

population closely following the high-growth scenario. The same cannot be said for the 1984-2006 and 1989-2011 editions (Figure 3): the unanticipated but considerable rise in immigration levels that occurred in the 1990s explains to a great extent why these projections ended up underestimating population growth. For mainly the same reason, these editions also overestimated the senior dependency ratio. ${ }^{7}$ In 2006, the actual ratio was 20.3. By comparison, for the same year, the 1984-2006 editions projected a senior dependency ratio between 24.1 and 25.2, while the 1989-2011 edition projected it to be between 22.1 and 24.7 .

Published after a period in which fertility and immigration had risen considerably, the 1993-2016 edition suggested a TFR of 1.7 children per woman and 250,000 immigrants per year under its mediumgrowth scenario-assumptions which are, interestingly, very similar to the assumptions utilized in the medium-growth scenario of the two latest population projections (2009-2036 and 2013-2063 editions). In the short term, fertility and immigration decreased from their previous levels, and so these projections ended up overestimating population growth while suggesting a younger age structure than what actually occurred. Still, the observed population beginning in 2004 is within the range provided by the low and medium-growth scenarios.

7. The 1984-2006 edition proposed total fertility rates of 1.4 and 2.2 in the low and high-growth scenarios, respectively. These respective values were 1.2 and 2.2 in the 1989-2011 edition. Fertility assumptions only begin to have an effect on the dependency ratio of seniors 18 years after the start of the projection, when the first births will reach the 18-64 age group. On the other hand, since most immigrants are in the prime working ages at their arrival in Canada, immigration tends to raise the share of the 18-64 age group in comparison to seniors (and thus lowers the senior dependency ratio, all other things being equal), at least over the course of the time horizons considered. 


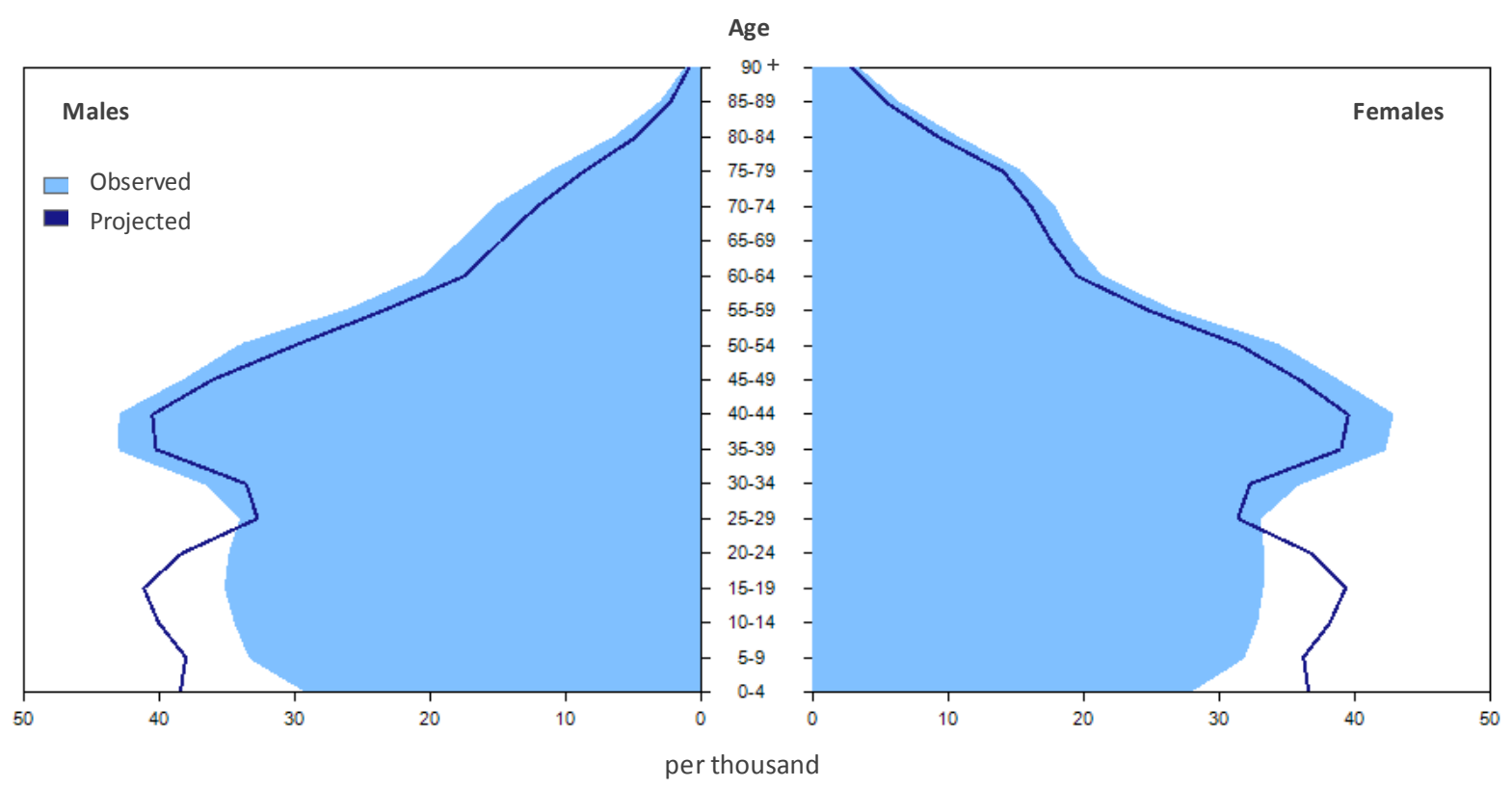

Figure 2. Age and sex pyramids (in relative value) of the Canadian population on 1 July 2001 according to the population projections of the 1972-2001 edition (medium growth scenario) and the population estimates.

Source: Statistics Canada, Demography Division. 2010. Population Projections for Canada, Provinces and Territories: 2009 to 2036. Chart 5.2.

Note: The projected figures were adjusted to take into account revisions made to the population estimates after the release of the projections. Essentially, these revisions consisted of adding net undercoverage and components that were not estimated previously such as non-permanent residents and returning emigrants.

A comparison of more recent editions of population projections with observed population levels to date must be made with caution, since it requires prejudging the accuracy of a long-term projection through its performance in the short term. Nevertheless, it remains a useful exercise, as it highlights recent demographic changes.

The 2000-2026 edition has underestimated population growth to date, as it projected that the population would reach 34.0 million in 2011 in the high-growth scenario-slightly less than the observed figure of 34.5 million. Multiple reasons explain this discrepancy. For one, this scenario has underestimated the number of births to date, projecting a linear increase in the TFR-from 1.54 in 1999 to reach 1.80 in 2026 — that has, so far, underestimated the observed trend. The scenario also underestimated the number of non-permanent residents, while it has overestimated the number of deaths. As a result of these assumptions, the projected age structure has also deviated slightly from what has been observed to date: while the observed senior dependency ratio (20.8) closely matches the values projected in 2011 by the medium and high-growth scenarios, the observed child dependency ratio (23.7) falls above the projected bracket, the closest of which was the high-growth scenario (23.4).

The 2005-2031 edition has also underestimated population growth to date, with the high-growth scenario projecting 34.2 million in 2011, compared to the 34.5 million observed in that year. The very small intervals between the various scenarios in the early years of the projection may partially explain why this has happened. Still, a combination of recent changes in Canadian demographics also contributed to this divergence, all of them entailing higher than expected growth: an interim rise in fertility, an increase in the number of immigrants and non-permanent residents, and a slight decrease in emigration levels. 


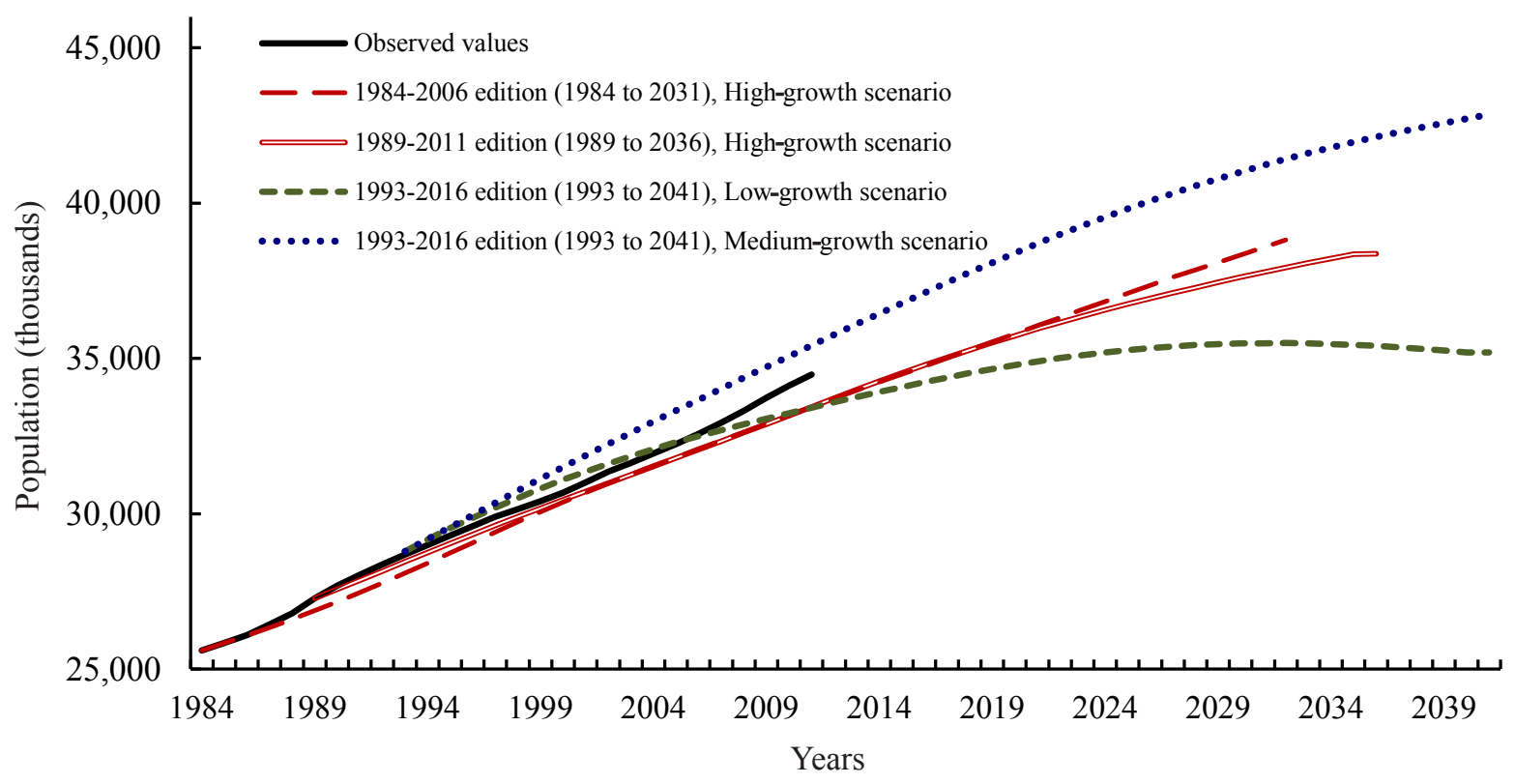

Figure 3. Population observed (1984 to 2011) and projected according to some scenarios taken from previous editions (1984-2006, 1989-2011 and 1993-2016), Canada.

Notes: Starting from the 1984-2006 edition, the horizon span of the projections was generally 20 years at the level of the provinces and the territories, and double that (40 years) at the national level. The official name of the edition continued to reflect the horizon span at the level of the provinces and territories.

The projected values in the 1984-2006 and 1989-2011 editions had, as a base point, a population unadjusted for the net undercoverage. To facilitate comparisons, in this figure, they have been adjusted to start at the same level as the observed values, which are adjusted for net undercoverage.

Finally, the most recent editions of the projections, those for the periods 2009-2036 and 20132063, remain at the present time out of scope for a thorough evaluation of accuracy. ${ }^{8}$ That said, at least in the short term, the steady declines in fertility that have occurred in Canada from 2008 to 2011 have occurred in stark contrast to the assumptions made for the 2009-2036 edition, which were formulated in the context of a period of strong gains in the total fertility rate from 2004 to 2007, suggesting that fertility may be the leading contributor to any errors in the short term.

The preceding analysis indicates that for the most part, past projections did capture generally the demographic growth and the key changes in the age structure of the national population, such as population aging, that have occurred since the 1970s (though the degree of population aging was underestimated). However, it also showed that some components of population growth displayed more discrepancies with historical values than others. This can be explained in part by the particular

8. The analysis contained in this article was completed prior to the release of the 2013-2063 edition. As explained previously, an evaluation of long-term population projections on the basis of short-term results should generally be avoided. In the case of the 2009-2036 edition, only two years of projections (2010 and 2011) could be made as of the time of writing of this article; while more recent data are now available, the latest final post-censal estimates of interprovincial migration are currently only available up to the period $2011 / 2012$. It is important to use final rather than preliminary estimates as a basis of evaluation, particularly in the case of interprovincial migration where substantial swings can occur between preliminary and final estimates.

9. The total fertility rate (TFR) increased from 1.53 in 2004 to 1.66 in 2007 , the latter value being the highest TFR observed since 1995. In the years following 2007, the TFR declined consecutively, reaching 1.61 in 2011. 
demographic context, in which some components may be more volatile than others, and the relative difficulties associated with building plausible assumptions in such contexts. ${ }^{10,11}$

At the national level, frequent underestimation of future levels of immigration explained why past projections most often suggested a deceleration of population growth that never materialized in the long term. This is not surprising: immigration is often reported as a common source of inaccuracy in population projections at the national level (e.g., Hollman et al. 2000; Statistics New Zealand 2008). In fact, compared to births or deaths, improvements in demographic science have not been of much help to the projection of immigration (Hollman et al. 2000). At least in the context of major immigrantreceiving countries such as Canada, the most important factor in terms of future levels of immigration turns out to be immigration policy. However, using levels prescribed by these policies may be satisfactory in the near future, but less so in the longer term (Hollman et al. 2000). Another difficulty is thatcontrary to other components-in the case of immigration, the at-risk population is by definition living in another country; therefore, it is usually not projected.

Past projections from Statistics Canada have established immigration assumptions using either predetermined numbers of immigrants or numbers evolving in proportion to the total population (immigration rates). The latter method was used in the two most recent editions, and implies that the demand for immigration would evolve in the future along with population growth. This may not be an unreasonable assumption for the next few decades, given the context in which baby boomers are slowly reaching the ages of retirement, leaving a smaller labour force in its wake. On the other hand, some unanticipated phenomenon could also easily prove these assumptions wrong-if, for instance, the number of immigrant admissions remained constant or decreased due to a deterioration of economic conditions in the country, if there is increased global competition for skilled immigrants, or if the capacity to integrate and support high levels of immigration is questioned. The complex interplay and feedback loops among the social, political, economic, and demographic contexts — within and outside of Canada — render it extremely difficult to formulate sound assumptions about immigration (Howe and Jackson 2004; Wilson and Rees 2005).

In contrast to immigration, past projections have shown more accuracy regarding mortality. The long-term trend of steady gains in life expectancy over the last century makes the formulation of assumptions less controversial. Demographers do not agree, however, as to whether an indefinite continuation of this trend can be expected in the more distant future. For instance, continuous scientific progress could lead to further gains in life expectancy, but new threats to health such as obesity, diabetes, pandemics, or antibiotic resistance could result in a stagnation or degradation of life expectancy (Bloom and Canning 2006). Additionally, while progress in life expectancy at birth has increased quite steadily over the past century, other aspects of mortality have been less predictable. For instance, the widening and subsequent closing of the gap in life expectancy between the sexes over the latter half of the 20th century could lead to various hypotheses of how mortality patterns by sex will evolve relative to one another over the long-term future.

Like the mortality component, the fertility component also exhibited trends that were more stable in the recent past in comparison to immigration. However, changes in fertility remain difficult to anticipate and may have a deep impact on the growth and the age structure of the population, as the analysis of the 1972-2001 edition showed. Small but sudden reversals in the trend of the total fertility

10. In line with what was stated earlier, from the perspective of population projections (and not population forecasts), a plausible assumption is not one that will necessary materialize, but one that, at the time of its conception, suggested a reasonable and realistic evolution given the existing knowledge.

11. Another aspect known for having an impact on accuracy, and documented in the context of this short review, is the time horizon. In general, accuracy tends to decrease with the time elapsed since the publication (George et al. 2004; Keilman 2007). 
rate are difficult to understand, as evidenced by the turnaround in formerly declining fertility rates in most developed countries in the late 2000s, followed by a return to declining trends experienced in many countries so far in the early 2010s (Goldstein et al. 2009, 2013; Bongaarts and Sobotka 2012). The complex relationship between structural changes in the magnitude of fertility, and the timing of births among consecutive cohorts of women, are noted by Booth (2006) as being the most difficult aspects of projecting fertility.

\section{Projections at the level of the provinces and territories}

Just as the accuracy of projections at the total population level may hide significant discrepancies within the age structure of the population, comparisons between observed and projected outcomes at the national level may conceal substantial variations at smaller geographic levels. In general, projections show more accurate results in larger places than in smaller places (George et al. 2004; Tayman and Swanson 1996). At the scale of the provinces and territories, growth depends on an additional component, internal migration (between provinces and/or territories), which can be the most important component of growth for some provinces and territories (Dion and Coulombe 2008). In general, we know that migration patterns between the provinces and territories are greatly influenced by fluctuating economic factors such as employment and wages (Coulombe 2006; Bernard et al. 2008); however, these factors are not easily predicted. For these reasons, Statistics Canada creates several distinct scenarios, in which only the internal migration assumptions vary, the goal being to reflect the higher uncertainty inherent to this component. Thus, when measuring the accuracy of past projections at the level of the provinces and territories, it is important to take into consideration the various internal migration scenarios.

As an illustrative example, we now proceed with an evaluation of the accuracy of the 2005-2031 projections at the level of the provinces and territories. Mindful of the aforementioned cautionary note of evaluating the accuracy of long-term projections through their performance in the short term, we aim here to instead highlight the general importance of internal migration assumptions, in terms of both growth and accuracy for the individual provinces and territories. A comprehensive evaluation would necessitate an examination of multiple editions.

It can be seen in Table 1 that for the medium-growth scenario, inaccuracy was generally higher at the scale of the provinces and territories than at the national level. Internal migration is an important explanatory factor in this phenomenon, as the wide range of outcomes from one scenario to the other denotes, but not the only one. Inaccuracies in the projected number of immigrants and emigrants will contribute divergence at the national level, but their distribution across the country-a factor that has evolved considerably in recent decades - is an additional potential source of inaccuracy at the level of provinces and territories. Similar to the compensating errors which occurred in the 1972-2001 edition already discussed, the differences in opposite signs at the levels of the provinces and territories can cancel out, leaving the national-level picture unaffected.

At the provincial and territorial levels, however, it appears that internal migration is usually the principal source of inaccuracy among all of the components of growth. Table 2 shows the mean annual absolute percentage errors measured for each component of growth in each province and territory in the 2005-2031 edition. Allowing for variation in the internal migration component, the scenario that best matched the observed data was selected in each province and territory, independently of all others. In spite of this procedure, internal migration appears to be the component that exhibits the worst performance in 5 of the 10 provinces and in the 3 territories. For instance, in the province 
Dion and Galbraith: A review of forty years of population projections at Statistics Canada

Table 1. Mean annual percent error of the annual growth over the 2005/2006 to 2010/2011 period, by province and territory, for the mediumgrowth scenarios in the 2005 to 2031 edition of population projections.

\begin{tabular}{lrrrr}
\hline \multirow{2}{*}{\multicolumn{1}{c}{ Province / Territory }} & Medium & $\begin{array}{c}\text { Recent } \\
\text { trends }\end{array}$ & West coast & $\begin{array}{c}\text { Central- } \\
\text { West }\end{array}$ \\
\cline { 2 - 6 } Newfoundland and Labrador & \multicolumn{4}{c}{$\%$} \\
\cline { 2 - 5 } Prince Edward Island & -0.01 & 0.20 & -0.17 & 0.65 \\
Nova Scotia & 0.56 & 0.64 & 0.52 & 0.72 \\
New Brunswick & -0.06 & 0.07 & -0.16 & 0.01 \\
Quebec & 0.05 & 0.18 & -0.08 & 0.17 \\
Ontario & 0.33 & 0.32 & 0.35 & 0.43 \\
Manitoba & 0.01 & -0.07 & 0.10 & -0.08 \\
Saskatchewan & 0.48 & 0.42 & 0.55 & 0.42 \\
Alberta & 1.25 & 1.29 & 1.23 & 0.90 \\
British Columbia & 1.11 & 0.81 & 1.41 & 0.65 \\
Yukon & 0.33 & 0.77 & -0.15 & 0.75 \\
Northwest Territories & 1.11 & 1.58 & 0.50 & 2.36 \\
Nunavut & -1.21 & -1.06 & -1.36 & 0.44 \\
CANADA & 1.00 & 0.47 & 1.43 & 0.90 \\
\hline
\end{tabular}

Notes: The mean per cent error is the average of the annual differences between the observed value and the projected figure divided by the observed population at the beginning of the year. Since the differences are not taken in absolute values, negative and positive differences for different years will cancel out in the calculation of the average. The averages are calculated over the period 2005/2006 to 2010/2011.

of Newfoundland and Labrador, in the most accurate of the four medium-growth scenarios (the recent-trends scenario), internal migration exhibited an annual percentage error of 0.39 per cent in absolute terms, which is much higher than the second-worst projected component, births $(0.09$ per cent). In other provinces, significant and sometimes unparalleled changes—such as variations in the number of new immigrants in Prince Edward Island and Ontario, the rise in the number of births in Québec, and the increase in the number of non-permanent residents in British Columbia-contributed to high levels of inaccuracy in components other than internal migration.

It should be noted that the time horizon for which the comparisons of projected and observed values are made may greatly influence the results; as mentioned earlier, several studies have found that discrepancies between projected and observed values increase with time (Rayer 2008). Given this, it could be expected that the internal migration component would show better accuracy in the short term, especially in the "recent trends" scenario, but this is not necessarily the case. Since migration tends to vary from one year to the next, the uncertainty associated with this component can be substantial in the short term. ${ }^{12}$ In contrast, other components, especially mortality and fertility, could be more accurate in the short term, since they tend to change much more gradually. However, preliminary sensitivity analyses of an older edition of the projections, the 1993-2016 edition, suggest that internal migration can be the largest contributor to inaccuracies for the provinces and territories in the longer term, as well. ${ }^{13}$

12. In the long term, these annual variations may cancel each other out - in which case, the global uncertainty related to internal migration would not increase very much over time.

13. The results, not shown here for economy of space, closely mimic the results obtained from the 2005-2031 edition, showing that internal migration was usually the principal source of inaccuracy among all of the 
Table 2. Mean annual absolute percent error measured for each component of growth over the 2005/2006 to 2010/2011 period, by province and territory, for the scenario showing the best match in the 2005 to 2031 edition of population projections.

\begin{tabular}{|c|c|c|c|c|c|c|c|c|c|}
\hline \multirow{2}{*}{$\begin{array}{l}\text { Province } \\
\text { /Territory }\end{array}$} & \multirow{2}{*}{ Scenario } & \multirow{2}{*}{ Births } & \multirow{2}{*}{ Deaths } & \multirow{2}{*}{$\begin{array}{l}\text { Immi- } \\
\text { gration }\end{array}$} & \multirow{2}{*}{$\begin{array}{c}\text { Total } \\
\text { emigration }\end{array}$} & \multirow{2}{*}{$\begin{array}{l}\text { Net change } \\
\text { in non- } \\
\text { permanent } \\
\text { residents }\end{array}$} & \multirow{2}{*}{$\begin{array}{c}\text { Net } \\
\text { internal } \\
\text { migration }\end{array}$} & \multicolumn{2}{|c|}{ Internal } \\
\hline & & & & & & & & $\begin{array}{l}\text { In-migra } \\
\text { tion rate }\end{array}$ & $\begin{array}{c}\text { Out-migra } \\
\text { tion rate }\end{array}$ \\
\hline & & & & & & $\%$ & & & \\
\hline N.L. & Recent trends & 0.09 & 0.03 & 0.03 & 0.02 & 0.05 & 0.39 & 0.12 & 0.31 \\
\hline P.E.I. & Central-West & 0.07 & 0.03 & 0.81 & 0.02 & 0.13 & 0.33 & 0.19 & 0.46 \\
\hline N.S. & Recent trends & 0.07 & 0.02 & 0.08 & 0.01 & 0.10 & 0.22 & 0.09 & 0.18 \\
\hline N.B. & Recent trends & 0.07 & 0.02 & 0.14 & 0.02 & 0.05 & 0.14 & 0.08 & 0.15 \\
\hline Que. & West coast & 0.14 & 0.07 & 0.07 & 0.01 & 0.08 & 0.04 & 0.08 & 0.07 \\
\hline Ont. & West coast & 0.06 & 0.03 & 0.11 & 0.01 & 0.07 & 0.04 & 0.10 & 0.12 \\
\hline Man. & Central-West & 0.08 & 0.02 & 0.35 & 0.04 & 0.05 & 0.11 & 0.21 & 0.17 \\
\hline Sask. & Central-West & 0.14 & 0.03 & 0.31 & 0.04 & 0.09 & 0.45 & 0.22 & 0.30 \\
\hline Alta. & Recent trends & 0.22 & 0.02 & 0.22 & 0.05 & 0.31 & 0.34 & 0.45 & 0.30 \\
\hline B.C. & Medium & 0.05 & 0.03 & 0.09 & 0.03 & 0.21 & 0.10 & 0.22 & 0.17 \\
\hline Y.T. & West coast & 0.02 & 0.08 & 0.28 & 0.03 & 0.20 & 0.53 & 2.54 & 2.55 \\
\hline N.W.T. & Central-West & 0.18 & 0.05 & 0.05 & 0.03 & 0.06 & 0.51 & 1.06 & 1.36 \\
\hline Nvt. & Recent trends & 0.07 & 0.04 & 0.04 & 0.04 & 0.05 & 0.40 & 0.26 & 0.49 \\
\hline
\end{tabular}

Notes : The values shown for a specific province or territory come from the scenario showing the best fit between observed estimates and projected values, among the four various medium-growth scenarios (each displaying a different internal migration assumption) of the 2005-2031 edition of population projection. The mean absolute per cent error is the average of the annual differences between the observed value and the projected value in absolute value, divided by the observed population at the beginning of the year. The averages are calculated over the period 2005/2006 to 2010/2011. The values in bold indicate the component where the highest values are found for a specific province or territory, thus indicating the component of growth where the highest average differences were found.

A second possible explanation as to why internal migration did not display better accuracy in the 2005-2031 edition relates to how the various assumptions were built. In this edition, outmigration rates by age and sex, from each origin to each destination specifically, were calculated for four alternative reference periods, each showing distinct patterns of net migration. The various internal migration assumptions were then created by averaging the out-migration rates in these various periods, and holding them constant throughout the projection. Since the projected internal migration flows depended only on population changes in the region of origin, and not in the region of destination, net migration tended to increase in regions losing demographic weight, and to decrease in regions gaining demographic weight. This purely mechanical effect is not only counterintuitive, but tended to impede the achievement of varied internal migration assumptions, which are crucial given the high volatility of this component over time. For this reason, the recently released edition of the population projections includes, among other innovations, a remedy to this methodological issue for internal migration that takes into account changes in the population sizes of the regions of destination, which result in more varied projected internal migration patterns for the individual provinces and territories across different scenarios (please see Dion 2014 and Bohnert et al. 2014b for more details).

components of growth for the provinces and territories (though the MAPE values were usually higher, likely in part due to a longer time horizon). Naturally, a more comprehensive evaluation of internal migration projection errors (beyond the scope of this paper) would require the analysis of several editions of population projections, various time horizons, and multiple indicators of accuracy. 


\section{Conclusion}

This article has aimed to act as a guide to users of Canadian population projections. More specifically, this article documents how Statistics Canada has, to date, approached the challenge of projecting the population. Success in projecting the future with accuracy is not only a matter of technique and assumptions; it is also largely a function of how stable the components of population growth have been, and will be in the years to follow.

Our review found that Statistics Canada's past projections successfully anticipated some important continuing phenomena, such as population growth and aging, but they also tended not to foresee changes, echoing a finding for the United States by the US Census Bureau (Mulder 2002). The unanticipated fertility declines in the 1980s, and the rise in immigration levels in the 1990s, explain why immigration and fertility assumptions have proved the least accurate at the national level. Likewise, at the level of the provinces and territories, changes in internal migration patterns are swift and very difficult to predict; internal migration assumptions appeared both particularly salient and likely to prove inaccurate.

The struggle to foresee change is perhaps to be expected; as Le Bras wrote: "Unavoidably, the projection assumptions reflect the spirit of the era in which they are framed. To them are transmitted its hopes and its fears" (2008: 153). This further emphasizes the importance of regarding projections as a series of "what if" scenarios-based on careful, informed analysis of trends in various demographic components as of the time of their production - and not as predictions, as forecasts aim to be. As per Romaniuc (2003), it is the plausibility of the assumptions more than the outcomes themselves that should be evaluated. We should refrain from concluding, therefore, that these previous projections contained assumptions that were implausible at the time, or that the projections were ineffective. Population projections, unlike population forecasts, were never meant to predict the future population growth and its composition, but rather to serve as planning and policymaking tools. When used as such, population projections may trigger changes that were unexpected, and therefore become the cause of their own eventual "inaccuracy." In the end, "[p]rojections are a success if they stimulate thought and action oriented toward creating a more desirable future" (Isserman 1992: 23). In other words, the utility of population projection is not in the future; it is in the present, as a tool to raise questions about the future.

As a final point, readers are invited to consult the Population Projections for Canada (2013 to 2063), Provinces and Territories (2013-2038), released in the fall of 2014 (Bohnert et al. 2014a). ${ }^{14}$ Following the previous edition, the projections team within the Demography Division conducted a comprehensive review of the National Population Projection Program, from which many possible improvements were identified. Consequently, this new edition contains many innovations, including: the incorporation of results from a survey of demography experts about their opinions on future demographic trends, separate fertility projection assumptions for non-permanent residents, as well as the previously mentioned new model for the projection of internal migration, among others. These new methodologies and innovations are described in detail in a distinct technical report ${ }^{15}$ accompanying the projections (Bohnert et al. 2014b). While none of these improvements can offset a sudden change in the components of population growth, taken together these new approaches have supported the construction of projection assumptions for the newest edition that are more transparent, intuitive, and provide a wide range of possible outcomes, representing the combined knowledge of experts and a comprehensive evaluation of trends.

14. See http://www.statcan.gc.ca/pub/91-520-x/91-520-x2014001-eng.htm. 15. See http://www.statcan.gc.ca/pub/91-620-x/91-620-x2014001-eng.htm. 


\section{Acknowledgements}

Some elements of this paper were presented at the 2012 annual meeting of the Population Association of America, in San Francisco. The authors would also like to thank Yvan Clermont, Laurent Martel, François Nault, Éric Caron Malenfant, and the two anonymous reviewers for their useful suggestions and comments in regards to this paper.

\section{References}

Beaujot, R. 2000. Canada's Demographic Future: Some Reflections on Projection Assumptions. Canada Pension Plan Seminar on Demographic and Economic Perspectives of Canada: Years 2000 to 2050. Ottawa: Office of the Chief Actuary.

Bernard, A., R. Finnie, and B. Saint-Jean. 2008. Interprovincial mobility and earnings. Perspectives on Labour and Income. (Cat. No. 75-001). Ottawa: Statistics Canada.

Bloom, D.E., and D. Canning. 2006. Global Demography: Fact, Force and Future. MPRA Paper 2577. Munich: University Library of Munich.

Bongaarts, J. and T. Sobotka. 2012. A demographic explanation for the recent rise in European fertility. Population and Development Review 38(1):83-120.

Bohnert N., J. Chagnon, S. Coulombe, P. Dion, and L. Martel. 2014a. Population Projections for Canada (2013 to 2063), Provinces and Territories (2013 to 2038). Cat. No. 91-520-X. Ottawa: Statistics Canada.

Bohnert N., J. Chagnon, and P. Dion. 2014b. Population Projections for Canada (2013 to 2063), Provinces and Territories (2013 to 2038): Technical Report on Methodology and Assumptions. Cat. No. 91-620-X. Ottawa: Statistics Canada.

Booth, H. 2006. Demographic forecasting: 1980 to 2005 in review. International Journal of Forecasting 22:547-81.

Coulombe, S. 2006. Internal migration, asymmetric shocks, and interprovincial economic adjustments in Canada. International Regional Science Review 29(2):199-223.

De Beer, J. 2000. Dealing with Uncertainty in Population Forecasting. Voorburg (Netherlands): Central Bureau of Statistics, Department of Population Statistics.

Dion, P. 2014. An alternative projection model for interprovincial migration in Canada. Proceedings of the Sixth Eurostat/UNECE Work Session on Demographic Projections. Rome: UNECE, p. 400-11.

Dion, P., and S. Coulombe. 2008. Portrait of the mobility of Canadians in 2006: Trajectories and characteristics of migrants. Report on the Demographic Situation in Canada, 2005 and 2006. Cat. No. 91 209-X. Ottawa: Statistics Canada.

George, M.V. 2001. Population forecasting in Canada: Conceptual and methodological developments. Canadian Studies in Population 28(1):111-54.

George, M.V., S.K. Smith, D.A. Swanson, and J. Tayman. 2004. Population projections, in The Methods and Materials of Demography, 2nd edn, edited by J.S. Siegel and D.A. Swanson. New York: Elsevier, p. 561-601.

Goldstein, J.R., T. Sobotka, and A. Jasilioniene. 2009. The end of "lowest-low" fertility? Population and Development Review 35(4):663-99. 
Dion and Galbraith: A review of forty years of population projections at Statistics Canada

Goldstein, J.R., M. Kreyenfeld, A. Jasilioniene, and K.D. Orsal. 2013. Fertility reactions to the 'Great Recession' in Europe: Recent evidence from order-specific data. Demographic Research 29(4):85-104.

Henry, L. 1972. Passé, présent et avenir en démographie. Population (French edition) 27(3): 383-96.

Hollmann F.W., T.J. Mulder, and J.E. Kallan. 2000. Methodology and Assumptions for the Population Projections of the United States: 1999 to 2100. Population Division Working Paper Series 38. Washington: US Census Bureau.

Howe, N., and R. Jackson. 2004. Projecting Immigration: A Survey of the Durrent State of Practice and Theory. Working Paper No. 2004-32. Boston: Boston College Center for Retirement Research.

Isserman, A. 1992. The Right People, the Right Rates: Making Population Estimates and Forecasts with an Interregional Cohort-Component Model. Research Paper 9216. Morgantown WV: West Virginia University.

Keilman, N. 2007. UK national population projections in perspective: How successful compared to those in other European countries? Population Trends 129:20-30.

Keyfitz, N. 1972. On future population. Journal of the American Statistical Association 67(338):347-63.

—. 1981. The limits of population forecasting. Population and Development Review 7(4):579-93. 1982. Can knowledge improve forecasts? Population and Development Review 8(44):729-51.

Keyfitz, N., and H. Caswell. 2005. Applied Mathematical Demography. 3rd edn. New York: Springer.

Lachapelle, R. 1977. Prévisions démographiques et processus de décision. Cabiers québécois de démographie 6(3):267-79.

Le Bras, H. 2008. The Nature of Demography. Princeton NJ: Princeton University Press.

Li, N., and Z. Wu. 2003. Forecasting cohort incomplete fertility: A method and an application. Population Studies 57(3):303-20.

Myrskyla, M., J. Goldstein, and Y-H.A. Cheng. 2013. New cohort fertility forecasts for the developed world: Rises, falls and reversals. Population and Development Review 39(1):31-56.

Mulder, T.J. 2002. Accuracy of the U.S. Census Bureau national population projections and their respective components of change. Population Division Working Paper Series 50. Washington: US Census Bureau.

O’Neill, B., D. Balk, M. Brickman, and M. Ezra. 2001. A guide to Global Population Projections. Demographic Research 4:203-88.

Plane, D.A. 1993. Requiem for the fixed-transition probability migrant. Geographical Analysis 25:211-23.

Preston, S. 1974. An evaluation of postwar mortality projections in Australia, Canada, Japan, New Zealand and the United States. WHO Statistical Report 27:719-45.

Rayer, S. 2008. Population forecast errors: A primer for planners. Journal of Planning Education and Research 27:417-30.

Romaniuc, A. 2003. Reflection on population forecasting: From predictions to prospective analysis. Canadian Studies in Population 30(1):35-50.

—. 2010. Population forecasting: Epistemological considerations. Genus 66(1):91-108.

Shaw, C. 2007. Fifty years of United Kingdom national population projections: How accurate have they been? Population Trends 128:8-23. 
Statistics Canada. 1974. Population Projections for Canada and the Provinces, 1972-2001. Cat. No. 91-514. Ottawa: Statistics Canada.

. 1980. Population Projections for Canada, Provinces and Territories 1976-2001. Cat. No. 91-520-X. Ottawa: Statistics Canada.

- 1985. Population Projections for Canada, Provinces and Territories 1984-2006. Cat. No. 91-520-X. Ottawa: Statistics Canada.

1990. Population Projections for Canada, Provinces and Territories 1989-2011. Cat. No. 91-520-X. Ottawa: Statistics Canada.

- 1994. Population Projections for Canada, Provinces and Territories 1993-2016. Cat. No. 91-520-X. Ottawa: Statistics Canada.

- 2001. Population Projections for Canada, Provinces and Territories 2000-2026. Cat. No. 91-520-X. Ottawa: Statistics Canada.

- 2005. Population Projections for Canada, Provinces and Territories 2005-2031. Cat. No. 91-520-X. Ottawa: Statistics Canada.

- 2010. Projections of the Diversity of the Canadian Population 2006 to 2031. Cat. No. 91-551-X. Ottawa: Statistics Canada.

- 2011. Population Projections by Aboriginal Identity in Canada 2006 to 2031. Cat. No. 91-552-X. Ottawa: Statistics Canada.

Statistics New Zealand. 2008. How Accurate are Population Projections? An Evaluation of Statistics New Zealand Population Projections, 1991-2006. Wellington: Statistics New Zealand.

Tayman, J., and D.A. Swanson. 1996. On the utility of population projections. Demography 33(4):523-28.

Werschler, T., and F. Nault. 1996. Projecting interregional migration balances within a multiregional cohort-component framework. Environment and Planning A 58:769-82.

Wilson, T., and P. Rees. 2005. Recent developments in population projection methodology: A review. Population, Space and Place 11:337-60.

Yokum, J.T., and J. S. Armstrong. 1995. Beyond accuracy: Comparison of criteria used to select forecasting methods. International Journal of Forecasting 11:591-97. 